\title{
Predictors, consequences and costs of diabetes-related lower extremity amputation complicating type 2 diabetes: The Fremantle Diabetes Study
}

\author{
W. A. Davis • P. E. Norman • D. G. Bruce • \\ T. M. E. Davis
}

Received: 2 March 2006 / Accepted: 31 July 2006 / Published online: 26 September 2006

(C) Springer-Verlag 2006

\begin{abstract} cohort of type 2 diabetic patients. 2000 .

Electronic supplementary material Supplementary material is available in the online version of this article at http://dx.doi.org/ 10.1007/s00125-006-0431-0 and is accessible to authorized users.

W. A. Davis $(\bowtie) \cdot$ D. G. Bruce $\cdot$ T. M. E. Davis

School of Medicine and Pharmacology,

University of Western Australia, Fremantle Hospital,

P.O. Box 480, Fremantle, WA 6959, Australia

e-mail: wdavis@cyllene.uwa.edu.au

\section{P. E. Norman}

School of Surgery and Pathology,

University of Western Australia, Fremantle Hospital,

Fremantle, WA, Australia
\end{abstract}

Aims/hypothesis The aims of this study were to assess the incidence, predictors, consequences, and inpatient cost of lower extremity amputation (LEA) in a community-based

Methods Between 1993 and 1996, 1,294 patients with type 2 diabetes were recruited to the longitudinal, observational Fremantle Diabetes Study. LEAs and mortality from cardiac causes were monitored until 30 June 2005. Inpatient costs (in A\$ in year 2000), derived using a case-mix approach, were available for the period from 1 July 1993 to 30 June

Results During follow-up 44 patients without LEA at baseline had a first-ever diabetes-related LEA, an incidence of 3.8 per 1,000 patient-years. Independent predictors of first-ever LEA included foot ulceration (hazard ratio [95\% CI]: 5.56 [1.24-25.01]), an ankle brachial index $\leq 0.90$ (2.21 [1.11-4.42]), $\mathrm{HbA}_{1 \mathrm{c}}$ (increase of 1\%: 1.30 [1.10 1.54]) and neuropathy (2.65 [1.30-5.44]). The risk of cardiac death was significantly increased in patients with LEA at baseline, although this was not an independent risk factor. The median (interquartile range) inpatient cost per
LEA admission was A $\$ 12,485$ (A \$6,037-A \$24,415), with a median length of stay of 24 (10-43) days.

Conclusions/interpretation First-ever LEAs in type 2 patients were associated with poor glycaemic control, foot ulceration and evidence of microvascular and macrovascular disease. Patients with LEA were at increased risk of cardiac death. LEAs contribute disproportionately to diabetes-related inpatient costs.

Keywords Cohort study · Cost · Epidemiology .

Lower extremity amputation · Type 2 diabetes

\author{
Abbreviations \\ ABI ankle brachial index \\ ACR urinary albumin:creatinine ratio \\ CVD cerebrovascular disease \\ DRG diagnostic related group \\ FDS Fremantle Diabetes Study \\ HR hazard ratio \\ ICD International Classification of Diseases \\ IQR interquartile range \\ LEA lower extremity amputation \\ LOS length of stay \\ PAD peripheral arterial disease \\ UKPDS United Kingdom Prospective Diabetes Study \\ WADLS Western Australian Data Linkage System
}

\section{Introduction}

Diabetes increases the risk of lower extremity amputation (LEA) by 10 to 20 times $[1,2]$ and is associated with half of all LEAs worldwide [3]. Apart from the obvious associated morbidity, diabetic patients with LEA have an 
increased risk of death from cardiovascular disease $[4,5]$. Vascular complications such as LEAs are also the major drivers of diabetes-related direct health care costs $[6,7]$. In the USA in 2001, the direct costs of inpatient care and prostheses for the estimated 42,424 diabetic patients undergoing LEA totalled US\$1.65 billion [8] or nearly $2 \%$ of the annual total direct health care cost of diabetes [9].

There is evidence that some of the complications of diabetes can be prevented or at least delayed costeffectively if glycaemia, serum lipids and blood pressure are treated intensively [10-12]. The role of this strategy in the prevention of LEA has yet to be confirmed. The United Kingdom Prospective Diabetes Study (UKPDS), which excluded patients with significant cardiovascular disease, did not have the statistical power to assess the effectiveness of intensive glycaemic control on LEA as a single complication [13]. Indeed, the non-significant 39\% reduction in the incidence of LEA in patients managed in this way probably reflected the relatively small number of such events [14].

In the light of the relative paucity of longitudinal data from well-characterised diabetic patients in the community, the aim of the present study was to assess the prevalence, incidence and predictors of LEAs in a large representative cohort. In addition, we estimated the direct inpatient costs of this important complication.

\section{Subjects and methods}

\section{Patients}

The Fremantle Diabetes Study (FDS) was a longitudinal observational study of patients from a postcode-defined community of 120,097 people. We identified 2,258 diabetic subjects between 1993 and 1996 using multiple sources (including hospital lists, general practitioners, specialists, allied health services, pharmacies and advertisements) and recruited $1,426(63 \%)$ to annual assessments, of whom $1,294(91 \%)$ had type 2 diabetes $[15,16]$. Eligible patients who declined participation were older than participants, but the proportion with type 2 diabetes and the distribution of treatment modalities were similar [17]. The FDS protocol was approved by the Human Rights Committee at Fremantle Hospital and all subjects gave informed consent before participation. The present sample comprised the 1,294 patients with type 2 diabetes who attended the baseline assessment.

\section{Clinical assessment}

All FDS patients were recruited between 1993 and 1996. At baseline and subsequent annual reviews, a comprehensive history of diabetes and co-morbid conditions was taken and a physical examination performed including detailed foot inspection $[15,16]$. A foot ulcer was considered present if there was one on either side. Abdominal obesity was defined as a waist circumference $\geq 102 \mathrm{~cm}$ in men and $\geq 88 \mathrm{~cm}$ in women [18]. Complications were identified using standard definitions. Peripheral sensory neuropathy was defined as a score of $>2 / 8$ on the clinical portion of the Michigan Neuropathy Screening Instrument [19]. A subject was considered to have retinopathy if any grade of retinopathy, including maculopathy, was detected by direct and/or indirect ophthalmoscopy in one or both eyes and/or on more detailed assessment by an ophthalmologist. Selfreported stroke and transient ischaemic attack were amalgamated with prior hospitalisations to define baseline cerebrovascular disease (CVD) status. Patients were classified as having CHD if there was a self-reported history of, or hospitalisation for, myocardial infarction, angina, coronary artery bypass grafting, angioplasty, and/or definite myocardial infarction on a Minnesota-coded electrocardiogram [20]. Peripheral arterial disease (PAD) was considered to be present when the ankle brachial index (ABI) was $\leq 0.90$ (either side) [21]. From automated biochemical analyses of fasting blood and urine samples provided by each patient, nephropathy was defined as a urinary albumin:creatinine ratio $(\mathrm{ACR}) \geq 3.0 \mathrm{mg} / \mathrm{mmol}$ in a first morning urine sample [22]. We calculated the estimated GFR using the Modification of Diet in Renal Disease equation [23].

Lower extremity amputations and mortality

A government register records details of all deaths and hospital admissions in Western Australia and is part of the larger Western Australian Data Linkage System (WADLS) [24]. The Confidentiality of Health Information Committee approved linkage with the FDS database. These sources provided hospital admissions and all-cause mortality from the beginning of the study until end-June 2005 . All hospital admissions for LEA were identified using relevant International Classification of Disease (ICD) procedure codes, i.e. ICD-9-CM and ICD-10-AM (see Electronic supplementary material). The corresponding diagnosis codes were examined to confirm that the LEAs were for diabetes-related reasons. The causes of death were reviewed independently by two of the authors (D. G. Bruce and T. M. E. Davis) and classified as being due to cardiac or other causes under the same system as used in the UKPDS [13]. The crude incidence of first-ever diabetesrelated LEA was determined in patients without LEA at study entry by dividing the number of first-ever diabetesrelated LEAs by the patient-years of follow-up to first-ever LEA or death or 30 June 2005 . The crude incidence of total 
diabetes-related LEAs was determined by dividing the number of all LEAs in the cohort during follow-up by the patient-years of follow-up to death or 30 June 2005. A major amputation was defined as through, or proximal to, the tarsometatarsal joint and a minor amputation as one distal to this joint [3].

Cost of hospitalisation for diabetes-related lower extremity amputation

Inpatient costs (in A $\$$ in year 2000) for the FDS type 2 cohort were derived using a case-mix approach based on Australian National Diagnosis Related Groups (AN-DRG) Version 3.1 (Commonwealth Department of Health and Family Services, Canberra, Australia) and were available for the period from 1 July 1993 to 30 June 2000 [22]. The Health Department of Western Australia provided 1999 to 2000 cost-weights for AN-DRG 3.1. Cost-weights are recomputed annually based on individually costed episodes [25] excluding accrual-type items such as superannuation, fringe-benefit tax and building depreciation.

Statistical analysis

The computer package SPSS for Windows (version 11.5) was used. Data are presented as proportions, means $( \pm \mathrm{SD}$ ), geometric mean (SD range), or, in the case of variables that did not conform to a normal or ln-normal distribution, median (interquartile range [IQR]). For independent samples, two-way comparisons for proportions were by Fisher's exact test, for normally distributed variables by Student's $t$ test, and for non-normally distributed variables by Mann-Whitney $U$ test. The nonparametric Kruskal-Wallis test was used to compare three or more independent non-normally distributed samples. A $p$ value $<0.05$ was considered significant. Survival (from LEA or cardiac mortality) curves defined by (1) sex, (2) FDS participation, and (3) baseline diabetes-related LEA status were constructed with the use of Kaplan-Meier estimates and compared with the log-rank test. Cox proportional hazards modelling (forward conditional variable entry and removal with $p<0.05$ and $>0.10$, respectively) was used to determine independent predictors of both first-ever LEA and cardiac mortality. All clinically plausible univariate variables with $p<0.20$ were considered for entry into the models, except, in the case of first-ever LEA, podiatrist visits, which were excluded since patients with ulcers were more likely to have attended a podiatrist. In the case of cardiac mortality, ABI, neuropathy, and BMI were excluded because it was not possible to measure these variables accurately in most of the baseline amputees. Exercise was also not considered since amputees may have been less likely to exercise because of LEA.

\section{Results}

\section{Amputations}

At baseline, 1.5\% (19/1,294) of patients had undergone prior LEA including four bilateral amputees. Of these, 15 (1.2\% $[95 \% \mathrm{CI}][0.7-2.0 \%])$ were diabetes-related, the remaining four being due to cancer (two), polio, and a motor vehicle accident. Of the 15 prevalent diabetes-related LEAs, ten were in men, but the difference in prevalence by sex was not significant $(p=0.20)$. During 11,775 patient-years of followup (mean $9.1 \pm 3.1$ years), there were a further 71 LEAs in 48 patients which were all diabetes-related, or $6.0 / 1,000$ patient-years. There were $40(56.3 \%)$ toe, $13(18.3 \%)$ below knee, 13 (18.3\%) above knee, three foot and two reamputations. Fifteen patients had multiple amputations with four progressing from toe to below knee amputations.

Of the 1,279 type 2 patients without diabetes-related LEA at baseline, 44 had their first-ever diabetes-related LEA during follow-up, an incidence of 3.8/1,000 patient-years. In men the incidence was $4.8 / 1,000$ patient-years compared with $2.9 / 1,000$ patient-years for women $(p=0.11$ by log-rank test). Twenty-seven had a first-ever minor amputation (2.3/1,000 patient-years). In the 1,284 subjects without a major diabetes-related LEA at baseline, 21 had a first-ever major LEA, an incidence of 1.8/1,000 patient-years.

The baseline univariate and independent determinants of incident first-ever diabetes-related LEA are shown in Tables 1 and 2, respectively. Patients who underwent a first-ever LEA during follow-up had longer diabetes duration, worse glycaemic control and higher systolic blood pressure, and hence were more likely to be insulin-treated and taking blood pressure-lowering medication, especially ACE inhibitors. They were also significantly more likely to have nephropathy, retinopathy and neuropathy, PAD and CVD. A higher proportion had foot ulcer(s) and had attended a podiatrist before study entry, although they were no more likely to have ever had diabetes education. Independent predictors of first-ever diabetes-related LEA were nephropathy, retinopathy, neuropathy, PAD and CVD, as well as poor glycaemic control. The presence of foot ulcer increased the risk of first-ever LEA fivefold.

The WADLS provided additional data relating to amputations in patients living in the catchment area but who were not recruited to the FDS. Between 1 January 1993 and 30 June 2005, there were 160 diabetes-related LEAs in 98 of all 2,258 diabetic patients identified in the study area. The likelihood of a first LEA during a mean follow-up of $10.2 \pm 3.6$ years was no different between recruited and non-recruited subjects ( 4.1 vs $4.8 \% ; p=0.13$ by log-rank test). After adjusting for age and sex using Cox proportional hazards modelling, non-recruited subjects were no more likely to experience LEA during follow-up than 
Table 1 Univariate baseline associates of first-ever diabetes-related LEA during follow-up

\begin{tabular}{|c|c|c|c|}
\hline Baseline variable & No diabetes-related LEA & Diabetes-related LEA & $p$ value \\
\hline$N$ & 1,235 & 44 & \\
\hline Age (years) & $63.9 \pm 11.4$ & $66.8 \pm 9.3$ & 0.050 \\
\hline Male $(\%)$ & 48.2 & 59.1 & 0.17 \\
\hline Diabetes duration (years) & $4.0(1.0-8.0)$ & $10.5(5.0-17.0)$ & $<0.001$ \\
\hline BMI $\left(\mathrm{kg} / \mathrm{m}^{2}\right)$ & $29.6 \pm 5.4$ & $29.1 \pm 5.2$ & 0.53 \\
\hline Abdominal obesity (\%) & 64.9 & 61.4 & 0.63 \\
\hline Fasting plasma glucose $(\mathrm{mmol} / \mathrm{l})$ & $8.4(6.9-10.7)$ & $10.7(7.8-12.4)$ & 0.002 \\
\hline $\mathrm{HbA}_{1 \mathrm{c}}(\%)$ & $7.4(6.4-8.8)$ & $8.8(7.0-10.7)$ & 0.001 \\
\hline $\mathrm{HbA}_{1 \mathrm{c}} \geq 7.0 \%(\%)$ & 62.0 & 77.3 & 0.040 \\
\hline \multicolumn{4}{|l|}{ Diabetic control (\%): } \\
\hline Diet & 33.2 & 4.5 & \multirow[t]{3}{*}{$<0.001$} \\
\hline OHAs & 56.1 & 59.1 & \\
\hline Insulin ( \pm OHAs) & 10.7 & 36.4 & \\
\hline Systolic blood pressure $(\mathrm{mmHg})$ & $150 \pm 24$ & $161 \pm 22$ & 0.004 \\
\hline Diastolic blood pressure $(\mathrm{mmHg})$ & $81 \pm 11$ & $79 \pm 11$ & 0.46 \\
\hline Pulse pressure $(\mathrm{mmHg})$ & $70 \pm 20$ & $81 \pm 18$ & $<0.001$ \\
\hline On blood pressure-lowering medication (\%) & 50.4 & 65.9 & 0.047 \\
\hline On ACE-inhibitors (\%) & 21.4 & 36.4 & 0.025 \\
\hline Total serum cholesterol (mmol/l) & $5.5 \pm 1.1$ & $5.7 \pm 1.1$ & 0.23 \\
\hline HDL-cholesterol (mmol/1) & $1.06 \pm 0.32$ & $1.10 \pm 0.31$ & 0.39 \\
\hline Serum triacylglycerol (mmol/l) & $1.9(1.1-3.3)^{\mathrm{a}}$ & $2.1(1.1-4.0)^{\mathrm{a}}$ & 0.27 \\
\hline On lipid-lowering medication (\%) & 10.5 & 9.1 & 1.00 \\
\hline On aspirin $(\%)$ & 21.7 & 25.0 & 0.58 \\
\hline Urinary albumin:creatinine ratio (mg/mmol) & $2.9(0.7-12.3)^{\mathrm{a}}$ & $9.4(1.9-46.1)^{\mathrm{a}}$ & $<0.001$ \\
\hline $\begin{array}{l}\text { Estimated GFR (MDRD; } \\
\mathrm{ml} \cdot \mathrm{min}^{-1} \cdot 1.73 \mathrm{~m}^{-2} \text { ) }\end{array}$ & $74.9(62.4-88.1)$ & $68.3(50.9-87.9)$ & 0.08 \\
\hline \multicolumn{4}{|l|}{ Chronic kidney disease stage $(\%)$ : } \\
\hline $1\left(\mathrm{GFR} \geq 90 \mathrm{ml} \cdot \mathrm{min}^{-1} \cdot 1.73 \mathrm{~m}^{-2}\right)$ & 22.0 & 20.5 & \multirow[t]{3}{*}{0.032} \\
\hline $2\left(60 \leq \mathrm{GFR}<90 \mathrm{ml} \cdot \mathrm{min}^{-1} \cdot 1.73 \mathrm{~m}^{-2}\right)$ & 57.7 & 43.2 & \\
\hline$\geq 3\left(\mathrm{GFR}<60 \mathrm{ml} \mathrm{min}^{-1} \cdot 1.73 \mathrm{~m}^{-2}\right)$ & 20.3 & 36.4 & \\
\hline Retinopathy, any (\%) & 14.8 & 52.5 & $<0.001$ \\
\hline Peripheral neuropathy (\%) & 29.6 & 60.5 & $<0.001$ \\
\hline Foot ulcer (\%; either side) & 1.0 & 6.8 & 0.015 \\
\hline Ankle:brachial index $\leq 0.90$ (\%; either side $)$ & 27.6 & 53.7 & 0.001 \\
\hline Cerebrovascular disease $(\%)$ & 9.1 & 25.0 & 0.002 \\
\hline $\mathrm{CHD}(\%)$ & 31.2 & 36.4 & 0.51 \\
\hline Any exercise in past 2 weeks (\%) & 72.6 & 70.5 & 0.73 \\
\hline \multicolumn{4}{|l|}{ Smoking status $(\%)$ : } \\
\hline Never & 45.1 & 38.6 & \multirow[t]{3}{*}{0.18} \\
\hline Ex- & 40.0 & 36.4 & \\
\hline Current & 14.8 & 25.0 & \\
\hline \multicolumn{4}{|l|}{ Alcohol consumption } \\
\hline (number of standard drinks per day) & $0(0-0.7)$ & $0(0-1.5)$ & 0.24 \\
\hline Ever attended a podiatrist (\%) & 29.5 & 59.1 & $<0.001$ \\
\hline Ever had diabetes education (\%) & 68.0 & 60.5 & 0.32 \\
\hline
\end{tabular}

Values are proportions, means $( \pm \mathrm{SD})$, geometric mean $(\mathrm{SD} \text { range })^{\mathrm{a}}$, or median (interquartile range [IQR])

$O H A$ oral hypoglycaemic agent

FDS participants (hazard ratio [HR] [95\% CI]: $1.41[0.94$ $2.12] ; p=0.10)$. Because we did not have access to clinical details such as LEA in non-recruited subjects prior to the FDS, it was not possible to compare prevalent LEA at the start of the FDS between non-recruits and participants.
Lower extremity amputation and mortality

Of the 15 FDS participants with diabetes-related LEA at study entry, $13(86.7 \%)$ died during follow-up compared with $434(33.9 \%)$ without $(p<0.001)$. Of these 15 partici- 
Table 2 Cox proportional hazards model of baseline predictors of time to first-ever diabetes-related LEA during follow-up

\begin{tabular}{lll}
\hline & Hazards ratio $(95 \% \mathrm{CI})$ & $p$ value \\
\hline History of cerebrovascular disease & $5.45(2.51-11.85)$ & $<0.001$ \\
$\mathrm{HbA}_{1 \mathrm{c}}$ (increase of $1 \%$ ) & $1.30(1.10-1.54)$ & 0.002 \\
Any retinopathy present $_{\text {Neuropathy present }}$ & $2.99(1.47-6.08)$ & 0.003 \\
Ln (urinary albumin:creatinine ratio) & $2.65(1.30-5.44)$ & 0.008 \\
Ankle:brachial index $\leq 0.90$ & $1.34(1.07-1.66)$ & 0.010 \\
Foot ulcer present (either foot) & $2.21(1.11-4.42)$ & 0.024 \\
\hline
\end{tabular}

${ }^{\text {a }}$ A 2.72 -fold increase in urinary albumin:creatinine ratio corresponds to an increase of 1 in $\ln$ (urinary albumin:creatinine ratio)

pants with diabetes-related LEA at study entry, 6 (40.0\%) had died from cardiac causes by 30 June 2005 , compared with $176(13.8 \%)$ deaths in participants without diabetesrelated LEA. The likelihood of cardiac death was significantly increased in patients with baseline LEA $(p<0.0001$, log-rank test). In Cox proportional hazards modelling, older age, male sex, higher $\ln (\mathrm{ACR})$, lower systolic blood pressure, the presence of retinopathy, CHD and CVD, and current smoking at baseline significantly predicted death from cardiac causes. After adjusting for these independent risk factors, the presence of diabetes-related LEA at baseline did not increase the risk of cardiac death significantly (HR [95\% CI]: 1.52 [0.59-3.92]; $p=0.39$ ).

Cost of hospitalisation for diabetes-related lower extremity amputation

Between study entry and 30 June 2000, there were 40 admissions (29 individuals) for diabetes-related LEAs, including revision. The total cost of these admissions was A $\$ 683,577$, or $9.3 \%$ of the total cost of diabetes-related hospital admissions during this period. This compared with 571 and 1,220 admissions for ischaemic heart disease and nephropathy, respectively, over the same period, with corresponding costs of $\mathrm{A} \$ 2.29$ million and $\mathrm{A} \$ 0.50$ million, or $31.2 \%$ and $6.8 \%$ of total diabetes-related hospital costs.

The median (IQR) inpatient cost per LEA admission was $\mathrm{A} \$ 12,485$ (A $\$ 6,037-\mathrm{A} \$ 24,415)$ (mean [range] A $\$ 17,089$ [A $\$ 1,679-A \$ 85,551])$, whilst the median length of stay (LOS) was 24 (10-43) days (mean 31 [2-193] days). Inpatient cost was strongly correlated with LOS (Spearman's rho $=0.98, p<0.001$ ), but not with age, sex, or site of LEA $(p \geq 0.10)$. No significant difference in LOS by admission year was observed (Kruskal-Wallis test, $p=0.41$ ).

\section{Discussion}

The present study is the largest in-depth longitudinal assessment of amputation complicating type 2 diabetes. Our data show that glycaemic control, foot ulceration, and evidence of microvascular disease, CVD and PAD are all independent predictors of incident LEAs. Patients with LEA at study entry were at increased risk of cardiac death, although LEA was not an independent predictor of this outcome. LEAs occurred infrequently during follow-up in the FDS cohort, but amputations (including toe amputations) were responsible for a disproportionate $9.3 \%$ of inpatient costs during this period.

Our baseline LEA prevalence of $1.2 \%$ in patients with type 2 diabetes is similar to the 1.3 (95\% CI: $0.6-2.0) \%$ found in a community-based study of 1,077 diabetic patients in the UK [26], but lower than the 4.9 (95\% CI: $3.7-6.4) \%$ reported in a predominantly male Veterans Affairs clinic-based study in the USA [27]. Our prevalence figure may be an underestimate as, due to their immobility, amputees may have been less likely to participate in the FDS. However, we have shown that there was no significant difference between FDS participants and nonparticipants in the incidence of LEA during follow-up, suggesting that the prevalence of diabetes-related LEA at FDS onset was representative of that in the Fremantle community.

The incidence rates of LEA reported in the literature vary considerably and available studies are difficult to compare due to differences in data sources, sample characteristics (particularly ethnicity), management (including approaches to the use of amputation in the management of the diabetic foot), adequacy of follow-up, and definitions of both numerator and denominator [28]. Reported incidence rates of diabetes-related LEAs range between $0.03 \%$ per year for Asians living in Leicestershire, UK, from 1980 to 1985 [29], to $1.80 \%$ per year in American Indians [30]. During a mean 9.1 years of follow-up from entry into the FDS, $3.4 \%$ of our patients had a first-ever LEA, representing a crude annual incidence of $0.4 \%$. This is towards the lower end of the range in published studies [27], but evidence for the benefits of intensive management of vascular risk factors has accumulated significantly since most of these studies were conducted, and the FDS incidence probably represents the consequence of contemporary clinical practice. The low incidence rates in the 
UKPDS $(0.10 \%$ per year in intensively treated patients and $0.16 \%$ in the conventionally treated group [14]) reflect the fact that the participants were relatively young, newly diagnosed and had low rates of pre-existing macrovascular disease.

The presence of a foot ulcer increased the risk of LEA nearly six-fold in our patients. Several other studies have reported an independent association between foot ulceration and subsequent LEA [27, 31] and the risk of LEA increases with the severity of ulceration [32]. Evidence of PAD $(\mathrm{ABI} \leq 0.9)$ was also an independent predictor of LEA, consistent with the findings of others [27, 33]. However, the most striking observation in the present study was that the three important manifestations of microvascular disease (nephropathy, retinopathy and neuropathy) were all independently associated with incident LEA. An association with neuropathy has been documented previously [27, 33] and, although neuropathy was not assessed in the Wisconsin Epidemiologic Study of Diabetic Retinopathy, a weak association with more severe retinopathy was observed (odds ratio for one step [95\% CI]: 1.07 [1.00-1.13]) [31].

There is evidence that skin oxygenation, muscle metabolism and wound healing are impaired by microcirculatory changes in the diabetic foot $[34,35]$. The risk of an ulcer and subsequent LEA increases when there is also loss of sensation and mechanical deformity associated with neuropathy and PAD [36]. Our data are, therefore, consistent with a plausible multifactorial causal pathway involving PAD, neuropathy and local microvascular changes in the foot $[34,36,37]$. As a likely reflection of diabetes-specific risk factors such as microangiopathy, we found that the incidence of first-ever minor amputations was higher than that of first-ever major amputations, in contrast to the findings reported for general populations [3].

The logical strategy to minimise the risk of an LEA would involve regular screening for neuropathy, PAD and early signs of mechanical foot problems [38, 39]. There is good evidence that simple patient foot care education can reduce ulcers and amputations [40]. Reiber et al. found that diabetic patients who did not attend outpatient diabetic education sessions were at increased risk of LEAs [33]. Intensified diabetic foot care can reduce amputations by $45 \%$ and minor operations by $38 \%$, with attendant reductions in both direct and indirect costs [41]. In our study, about $40 \%$ of those who had a first LEA during follow-up had never attended diabetes education sessions or seen a podiatrist prior to study entry, and $16 \%$ had attended neither.

We also found that that poor glycaemic control was an independent risk factor for LEA. For each $1 \%$ increase in $\mathrm{HbA}_{1 \mathrm{c}}$ at baseline in FDS type 2 patients, there was an associated $30 \%$ increased risk of first LEA during followup. This is consistent with several studies showing that the risk of amputation increases with levels of $\mathrm{HbA}_{1 \mathrm{c}}$ [31, 42]. In the UKPDS, relatively intensive blood glucose control failed to reduce the incidence of LEAs significantly. Since older patients ( $>65$ years) and those with known CHD were excluded, the UKPDS may have been underpowered for LEA as an outcome, as there was a trend towards benefit in the intensive vs conventional protocol group (relative risk [95\% CI]: 0.61 [0.28-1.33]) [14]. The Diabetes Control and Complications Trial in type 1 diabetes found that intensive management reduced microvascular complications relative to conventional treatment [43] and, in a Monte Carlo simulation model of lifetime costs and benefits, intensively managed patients had 5.6 more years free from amputation [44]. Our data also suggest that improved glycaemic control should help reduce the risk of LEA complicating diabetes. Unfortunately, consistent with the fact that most Australian diabetic patients do not have proactive intensification of treatment to maintain a $\mathrm{HbA}_{1 \mathrm{c}}<7.0 \%$ [45], three-quarters of the FDS patients undergoing LEA had an $\mathrm{HbA}_{1 \mathrm{c}}$ above this level.

Hospital-based cohort studies of diabetic patients report that foot ulceration or LEA doubles the risk of death at 5 years $[4,5,46]$. In the present study, diabetic patients with LEA at entry were significantly more likely to die from cardiac causes during follow-up. However, we did not find that LEA was an independent predictor of cardiac mortality. This is consistent with other studies and is probably due to an association with older age and other manifestations of cardiovascular disease, notably CHD and CVD [5].

The average hospital cost of an admission for a diabetesrelated LEA in the current study was $\mathrm{A} \$ 17,089$ (in $\mathrm{A} \$$ in year 2000) with an average LOS of 31 days. In one Dutch study, the average total direct cost of a diabetes-related LEA, excluding rehabilitation, was $£ 10,531$ (pounds sterling) in 1992 (or A $\$ 26,810$ using the exchange rate for that year) with a mean hospital stay of 42 days [47]. The higher costs and longer LOS found in the Dutch study may be due to the different healthcare systems and the trend to earlier discharge from hospital in recent years. An earlier Australian study reported a shorter average LOS of 25 bed-days for diabetes-related LEAs [48]. The difference is likely to be due to the different methodologies, in that the earlier Australian study used aggregate data (the Australian National Hospital Morbidity Database) with the limitation that patients were identified with diabetes by ICD-9CM diagnosis codes on discharge records and therefore also included younger patients with type 1 diabetes. In the FDS, type 1 patients admitted for LEA between January 1993 and June 2005 had an average LOS of only 19 days. Nevertheless, overall these studies concur that diabetesrelated LEAs are very costly. The FDS has also shown that nearly 1 in 10 dollars spent on diabetes-related hospitalisations are for LEAs. 
One strength of the FDS is that there is a low rate of migration from Western Australia to other Australian states and overseas [49]. Of the baseline FDS cohort, 1,237 patients or $96 \%$ had been captured on the WADLS by endJune 2005. In addition, we contacted surviving participants every year to arrange annual assessment and 27 of the 57 non-captured subjects were reviewed in 2000 or 2001. We conservatively assumed that non-captured patients did not have an LEA during follow-up and were still alive at study end. A limitation of the present data is that most $(82 \%)$ of the first-ever LEAs occurred after FDS close-out in November 2001, so we were unable to corroborate the hospital morbidity database coding for LEA with foot examination by FDS staff. However, an audit of the WADLS with medical records for carotid endarterectomy in the general population revealed no coding errors (false positives) and only $1.3 \%$ missing cases (false negatives) [50]. Despite an average 9 years of follow-up, the present study was not powered to detect a significant sex-related difference in the incidence of first-ever LEA. However, we did find that 1.7 times more males than females had a firstever LEA during follow-up, in agreement with findings in general populations [3]. A limitation of using DRG costs is that they represent an average cost of all patients with the condition(s) for which the hospitalisation occurred, and may therefore underestimate the true cost of hospitalisation for a diabetic patient.

Amputation, which is potentially preventable, results in substantial morbidity, and is associated with increased mortality and considerable cost. Diabetic patients identified as being at increased risk of LEA, notably those with foot ulceration, PAD or microvascular complications, should be targeted for aggressive risk factor management, in particular glycaemic control and foot care.

Acknowledgements We thank the FDS patients for their participation. We are grateful to FDS staff for help with collecting and recording clinical information. We thank the Biochemistry Department at Fremantle Hospital and Health Service for performing laboratory tests, and the Diabetic Education, Podiatry and Dietetic Departments for assistance with recruitment of patients. The Raine Foundation, University of Western Australia funded the FDS. W. A. Davis had full access to all the data in the study and takes responsibility for the integrity of the data and the accuracy of the data analysis.

\section{References}

1. van Houtum WH, Lavery LA (1996) Outcomes associated with diabetes-related amputations in the Netherlands and in the state of California, USA. J Intern Med 240:227-231

2. Wrobel JS, Mayfield JA, Reiber GE (2001) Geographic variation of lower-extremity major amputation in individuals with and without diabetes in the Medicare population. Diabetes Care $24: 860-864$
3. The Global Lower Extremity Amputation Study Group (2000) Epidemiology of lower extremity amputation in centres in Europe, North America and East Asia. Br J Surg 87:328-337

4. Apelqvist J, Larsson J, Agardh C-D (1993) Long-term prognosis for diabetic patients with foot ulcers. J Intern Med 233:485-491

5. Faglia E, Favales F, Morabito A (2001) New ulceration, new major amputation and survival rates in diabetic subjects hospitalised for foot ulceration from 1990 to 1993. Diabetes Care 24: $78-83$

6. Jonsson B (2002) Revealing the cost of Type II diabetes in Europe. Diabetologia 45:S5-S12

7. Colagiuri S, Colagiuri R, Conway B, Grainger D, Davey P (2003) DiabCost Australia. Assessing the burden of type 2 diabetes in Australia. Diabetes Australia, Canberra

8. Gordois A, Scuffham P, Shearer A, Oglesby A, Tobian JA (2003) The health care cost of diabetic peripheral neuropathy in the US. Diabetes Care 26:1790-1795

9. American Diabetes Association (2003) Economic costs of diabetes in the US in 2002. Diabetes Care 26:917-932

10. Gray A, Raikou M, McGuire A et al (2000) Cost effectiveness of an intensive blood glucose control policy in patients with type 2 diabetes: economic analysis alongside randomised control trial (UKPDS 41). Br Med J 320:1373-1378

11. Jonsson B, Cook JR, Pedersen TR (1999) The cost-effectiveness of lipid lowering in patients with diabetes: results from the $4 \mathrm{~S}$ trial. Diabetologia 42:1293-1301

12. UK Prospective Diabetes Study Group (1998) Cost effectiveness analysis of improved blood pressure control in hypertensive patients with type 2 diabetes: UKPDS 40. Br Med J 317:720-726

13. UK Prospective Diabetes Study (UKPDS) (1991) VIII. Study design, progress and performance. Diabetologia 34:877-890

14. UK Prospective Diabetes Study Group (1998) Intensive bloodglucose control with sulphonylureas or insulin compared with conventional treatment and risk of complications in patients with type 2 diabetes (UKPDS 33). Lancet 352:837-853

15. Davis T, Zimmet P, Davis W, Bruce D, Fida S, Mackay I (2000) Autoantibodies to glutamic acid decarboxylase in diabetic patients from a multiethnic Australian community: the Fremantle Diabetes Study. Diabet Med 17:667-674

16. Bruce DG, Davis WA, Davis TME (2000) Glycemic control in elderly subjects with type 2 diabetes mellitus in the Fremantle Diabetes Study. J Am Geriatr Soc 48:1449-1453

17. Norman PE, Davis WA, Bruce DG, Davis TME (2006) Peripheral arterial disease and risk of cardiac death in type 2 diabetes: The Fremantle Diabetes Study. Diabetes Care 29:575-580

18. World Health Organisation (1998) Obesity: preventing and managing the global epidemic. Report of a WHO Expert Committee. WHO, Geneva

19. Feldman E, Stevens M, Thomas P, Brown M, Canal N, Greene D (1994) A practical two-step quantitative clinical and electrophysiological assessment for the diagnosis and staging of diabetic neuropathy. Diabetes Care 17:1281-1289

20. Davis TME, Fortun P, Mulder J, Davis WA, Bruce DG (2004) Silent myocardial infarction and its prognosis in a communitybased cohort of diabetic patients: The Fremantle Diabetes Study. Diabetologia 47:395-399

21. Leng GC, Fowkes FGR, Lee AJ, Dunbar J, Housley E, Ruckley CV (1996) Use of ankle brachial pressure index to predict cardiovascular events and death: a cohort study. $\mathrm{Br}$ Med J 313:1440-1444

22. Davis WA, Knuiman MW, Hendrie D, Davis TME (2005) Determinants of diabetes-attributable non-blood glucose-lowering medication costs for type 2 diabetes: The Fremantle Diabetes Study. Diabetes Care 28:329-336

23. Levey AS, Bosch JP, Lewis JB, Greene T, Rogers N, Roth D (1999) A more accurate method to estimate glomerular filtration 
rate from serum creatinine: a new prediction equation. Modification of Diet in Renal Disease Study Group. Ann Intern Med 130:461-470

24. Holman CD, Bass AJ, Rouse IL, Hobbs MS (1999) Populationbased linkage of health records in Western Australia: development of a health services research linked database. Aust N Z J Public Health 23:453-459

25. Hendrie D, Boldy D (2002) Hospital services in Western Australia. Aust Health Rev 25:173-188

26. Walters DP, Gatling W, Mullee MA, Hill RD (1992) The distribution and severity of diabetic foot disease: a community study with comparison to a non-diabetic group. Diabet Med 9:354-358

27. Adler AI, Boyko EJ, Ahroni JH, Smith DG (1999) Lowerextremity amputation in diabetes. The independent effects of peripheral vascular disease, sensory neuropathy, and foot ulcers. Diabetes Care 22:1029-1035

28. Jeffcoate WJ, van Houtum WH (2004) Amputation as a marker of the quality of foot care in diabetes. Diabetologia 47:20512058

29. Gujral JS, McNally PG, O’Malley BP, Burden AC (1993) Ethnic differences in the incidence of lower extremity amputation secondary to diabetes mellitus. Diabet Med 10:271-274

30. Lee JS, Lu M, Lee VS, Russell D, Bahr C, Lee ET (1993) Lowerextremity amputation. Incidence, risk factors, and mortality in the Oklahoma Indian Diabetes Study. Diabetes 42:876-882

31. Moss SE, Klein R, Klein BEK (1999) The 14-year incidence of lower-extremity amputations in a diabetic population. The Wisconsin Epidemiologic Study of Diabetic Retinopathy. Diabetes Care 22:951-959

32. Margolis DJ, Allen-Taylor L, Hoffstad O, Berlin JA (2005) Diabetic neuropathic foot ulcers and amputation. Wound Repair Regen 13:230-236

33. Reiber GE, Pecoraro RE, Koepsell TD (1992) Risk factors for amputation in patients with diabetes mellitus. Ann Intern Med 117:97-105

34. Greenman RL, Panasyuk S, Wang X et al (2005) Early changes in the skin microcirculation and muscle metabolism of the diabetic foot. Lancet 633:1711-1717

35. Falanga V (2005) Wound healing and its impairment in the diabetic foot. Lancet 366:1736-1743

36. Cavanagh PR, Lipsky BA, Bradbury AW, Botek G (2005) Treatment for diabetic foot ulcers. Lancet 366:1725-1735
37. Abbott CA, Carrington AL, Ashe H et al (2002) The North-West Diabetes Foot Care Study: incidence of, and risk factors for, new diabetic foot ulceration in a community-based patient cohort. Diabet Med 19:377-384

38. American Diabetes Association (2003) Peripheral arterial disease in people with diabetes. Diabetes Care 26:3333-3341

39. Mayfield JA, Reiber GE, Sanders LJ, Janisse D, Pogach LM, American Diabetes Association (2003) Preventive foot care in people with diabetes. Diabetes Care 26:S78-S79

40. Malone JM, Snyder M, Anderson G, Bernhard VM, Holloway GA, Bunt TJ (1989) Prevention of amputation by diabetic education. Am J Surg 158:520-523

41. Edmonds ME, Blundell MP, Morris ME, Thomas EM, Cotton LT, Watkins PJ (1986) Improved survival of the diabetic foot: the role of a specialised foot clinic. Q J Med 60:763-771

42. Stratton IM, Adler AI, Neil HAW et al (2000) Association of glycaemia with macrovascular and microvascular complications of type 2 diabetes (UKPDS 35): prospective observational study. Br Med J 321:405-412

43. DCCT Group (1993) The effect of intensive treatment of diabetes on the development and progression of long-term complications in insulin-dependent diabetes mellitus. N Engl J Med 329:977-986

44. DCCT Research Group (1996) Lifetime benefits and costs of intensive therapy as practiced in the diabetes control and complications trial. JAMA 276:1409-1415

45. Davis TME, Davis WA, Bruce DG (2006) Glycaemic levels triggering therapeutic intensification in type 2 diabetes in the community: The Fremantle Diabetes Study. Med J Aust 184:325328

46. Boyko EJ, Ahroni JH, Smith DG, Davignon D (1996) Increased mortality associated with diabetic foot ulcer. Diabet Med 13:967972

47. van Houtum WH, Lavery LA, Harkless LB (1995) The cost of diabetes-related lower extremity amputations in the Netherlands. Diabet Med 12:777-781

48. Payne CB (2000) Diabetes-related lower-limb amputations in Australia. Med J Aust 173:352-354

49. Bradshaw PJ, Jamrozik K, Jelfs P, Le M (2000) Mobile Australians: a moving target for epidemiologists. (letter) Med J Aust 172:566

50. Norman PE, Semmens JB, Laurvick CL, Lawrence-Brown M (2003) Long-term relative survival in elderly patients after carotid endarterectomy: a population-based study. Stroke 34:95-98 